\title{
Filariasis elimination, vector control and eradication challenges
}

\section{Commentary on Webber, R. Eradication of Wuchereria bancrofti infection through vector control. Trans Roy Soc Trop Med and Hyg 1979;73:722-4}

\author{
David H. Molyneux* \\ Department of Parasitology, Liverpool School of Tropical Medicine, Pembroke Place, Liverpool, L3 5QA, UK \\ *Corresponding author: E-mail: david.molyneux@lstmed.ac.uk
}

Received 19 June 2018; revised 5 July 2018; editorial decision 5 July 2018; accepted 6 July 2018

Keywords: Anopheles, elimination, eradication, filariasis, malaria, neglected tropical diseases, Wuchereria bancrofti

The selection of this paper from 1979 in the Transactions of the Royal Society of Tropical Medicine and Hygiene highlights significant issues in relation to neglected tropical disease (NTD) programmes, specifically the Global Programme to Eliminate Lymphatic Filariasis (LF), and the wider concepts of eradication and elimination. Webber in other papers in the 1970s described the epidemiology of Wuchereria bancrofti filariasis in the Solomon Islands where $W$. bancrofti and $P$. falciparum malaria were co-endemic, both transmitted by Anopheles punctulatus complex mosquitoes. The Solomon Islands were amongst the most endemic countries for filariasis in the Pacific region. ${ }^{1} \mathrm{~A}$ malaria eradication programme had been initiated in 1968, based on 6-monthly indoor residual spraying (IRS) of DDT (see papers quoted in Webber 1979). ${ }^{2}$ Webber's paper points out the significant impact of vector control on the parasitological parameters of W. bancrofti and, thus, the prospect of 'eradication' where Anopheles were vectors, in contrast to other areas of the Pacific where W. bancrofti was transmitted by Aedes. ${ }^{3}$ Webber comments perceptively that the degree of reduction in Anopheles vectors that is required is much less than that to control malaria, a fact particularly pertinent to African settings highlighted by ${ }^{4}$ and confirmed in Nigeria, ${ }^{5}$ where both impregnated bed nets, long-lasting impregnated nets and mass drug administration were deployed together. This resulted in the Federal Ministry of Health of Nigeria recommending greater coordination between the malaria and filariasis programmes in that country.

Furthermore, a recent study in the Gambia where filariasis was historically endemic, with high prevalences ${ }^{6}$ showed a decline in prevalence over a 30-year period as a result of Anopheles control, using bed nets to control malaria in a nationwide programme, ${ }^{7}$ although no mass drug administration (MDA) was implemented to control filariasis. This study demonstrated that vector control alone could sustainably arrest $W$. bancrofti transmission and lead to national elimination in an
Africa setting, ${ }^{8}$ and also emphasized the importance of the use of long-lasting impregnated nets to complement MDA in national Filariasis programmes borne out by recent results from Zambia. ${ }^{9}$ Webber's results in the Solomon islands clearly show the rate of decline in the parasitological parameters from $22 \%$ prevalence in 1974 to $\%$ prevalence in 1977 when no drugs were used in LF control. Webber was also able to estimate from his studies the longevity of adult $W$. bancrofti of 7-12 years, an important figure in the context of the current LF programmes dependent on MDA alone. At least 5 years of annual MDA is recommended by $\mathrm{WHO}$; given that the drugs are microfilaricidal and do not kill adult worms (macrofilaricidal) it could be expected that after 7 years MDA viable adults will still be present and producing microfilaria, which could sustain transmission. This could explain the persistent transmission in areas that have received more than 10 years MDA-so-called hotspots. In this context the Onchocerciasis Control Programme (OCP) in West Africa was initially scheduled to use a strategy of weekly larvicidal treatment for a period of 20 years, which was based on the estimated duration of adult Onchocerca volvulus worm life. However, after several years of vector control had been deployed it became clear from epidemiological studies that the estimated maximum longevity of adult Onchocerca was some 14 years; the advent of ivermectin into the OCP further reduced the estimated duration of combined MDA and vector control by some 2 years. ${ }^{10}$

The other feature of the paper ${ }^{2}$ is the use of the term eradication to describe the impact of indoor residual spraying on filariasis in the Solomon Islands. The paper was published during the final stages of the global smallpox eradication programme, which was formally declared to be eradicated by the World Health Assembly in 1980 - the only infection to be eradicated to date-WHO having abandoned the global malaria eradication programme in 1970 for reasons related to the development of DDT and chloroquine resistance. Today, the

(c) The Author(s) 2018. Published by Oxford University Press on behalf of Royal Society of Tropical Medicine and Hygiene. All rights reserved. For permissions, please e-mail: journals.permissions@oup.com. 
term that should have been used is 'elimination', given the current widely accepted definitions. In 1993, the International Task Force for Disease Eradication had evaluated around 90 candidate diseases and concluded that six were eradicablemumps, rubella, lymphatic filariasis, cysticercosis, dracunculiasis and polio. Seminal books on eradication have since been published $^{11,12}$ and three diseases have been targeted by WHO for eradication-poliomyelitis, dracunculiasis and yaws, the first two diseases with active and highly successful programmes, which have yet to achieve the ultimate goal defined by WHO of the 'Permanent reduction to zero of the worldwide incidence of infection caused by a specific agent as a result of deliberate efforts. Intervention measures are no longer needed.' The challenges to achieving the goals of either elimination or, indeed, eradication have been highlighted. ${ }^{13,14}$ However, as has been pointed out, ${ }^{13}$ elimination in island settings is more easily achieved than when infections are on a continental or regional scale, where borders require greater coordination between countries and ${ }^{15}$ enhanced surveillance of vector migration (e.g. Simulium movement in West Africa) and population movement carrying infections. Islands provide a limited likelihood of vector reinvasion; a recent example is the successful blackfly control by larvicidal treatment, which eliminated transmission of onchocerciasis in Bioko, Equitorial Guinea. ${ }^{16}$ Another example of successful island elimination is of Echinococcus granulosus (hydatid disease) in Iceland, Tasmania, Cyprus, the Falkland Islands and New Zealand. ${ }^{17}$ Isolated foci of $O$. volvulus in Uganda have also been eliminated (where there was no risk of blackfly reinvasion) by vector control where S. neavei was the vector. ${ }^{18}$

The use of the term eradication by Webber in the title is excusable, but brings to attention the fact that, while elimination in island settings is feasible, the global goals of permanent zero incidence of any specific pathogen certified for all countries is an expensive journey that presents a huge challenge when seeking to 'prove a negative' in some of the most inaccessible, resource poor and conflict affected parts of the world. ${ }^{13,19}$ Consistency in the use of the definitions of eradication and elimination is also necessary, but it seems that even public health practitioners find this difficult to adhere to. ${ }^{20}$ These problems are also compounded by World Health Assembly resolutions that use the term 'as a public health problem', which are frequently based on parameters that are difficult to define and evaluate.

Roger Webber's work in the Solomon Islands deserves to be recalled given the success to-date of the Global Filariasis Programme. $^{21}$ This is because the value of vector control as an effective way of stopping transmission in Anopheles driven settings has been downplayed, in particular in African settings, where parallel malaria vector control will have had a synergistic impact where MDA has been deployed. ${ }^{8}$ Filariasis and other NTD MDA programmes should be linked to better resourced programmes. However, there has been a relative neglect of the opportunities afforded by vector or intermediate host control interventions as MDA programmes for NTDs have expanded. The fundamental fact is that transmission is generated by exposure to infected vectors or contact with cercariae-infected water or copepod ingestion in the case of Guinea worm, where copepod control has been an important intervention as part of a multifaceted complement of the Guinea Worm Eradication programme. ${ }^{14}$ While elimination has been successful in some settings without vector control, as in the Onchocerciasis Control Programme in the Americas leading to verified cessation of transmission in four countries (Colombia, Ecuador, Guatemala, Mexico), the duration of twice a year ivermectin MDA could have been foreshortened had vector control been feasible.

The use of 'eradication' in Webber's title (more correctly elimination) raises the challenges of what eradication really means. Eradication defined as 'zero global incidence of a specific infectious agent' needs a 'buyer beware' notice and reality-check warning. The current experience of polio and dracunculiasis programmes demonstrate that the last mile is proving remarkably difficult. Both polio and Guinea worm are recording a handful of human cases each year, in some of the countries where it is most difficult to implement programmes and access populations. In addition, a recently confirmed human case of Guinea worm infection in Angola, close to the Namibian border, is cause for serious concern, given that this case is so distant from the nearest remaining endemic areas in Chad, Ethiopia and Mali. ${ }^{22}$ This unexpected event needs urgent investigation, while the emergence of previously unsuspected animal hosts (dogs and baboons) of Dracunculus medinensis should temper expectations that zero global incidence of the pathogen can be proven - 'expect the unexpected' as remarked by the Dahlem eradication meeting. ${ }^{12}$ Webber's work on filariasis benefitted from the malaria control programme. Malaria eradication is now on the agenda. However, malaria 'eradication' itself does not comply with the accepted definition as malaria is a disease; the causative agents are the six human species of Plasmodium-two of which are proven zoonoses in primates. For 'malaria eradication' to be achieved each species of human Plasmodium must be confirmed as having zero global incidence. Perhaps it is time to consider a serious reality check?

Authors' contributions: DHM is the sole author; he selected the paper for comment, developed the ideas and wrote the paper.

Acknowledgements: None.

Funding: None.

Competing interests: None declared.

Ethical approval: Not required.

\section{References}

1 Ichimori K, Graves P. Overview of PacELF-the Pacific Programme for the Elimination of Lymphatic Filariasis. Trop Med Health 2017;45:34.

2 Webber R. Eradication of Wuchereria bancrofti infection through vector control. Trans R Soc Trop Med Hyg 1979;73:722-4. 


\section{H. Molyneux}

3 Pichon G. Limitation and facilitation in the vectors and other aspects of the dynamics of filarial transmission: the need for vector control against Anopheles transmitted filariasis. Ann Trop Med Parasitol 2002;96(Suppl 2):S143-52.

4 Molyneux DH, Nantulya V. Linking disease control programmes in rural Africa: a pro-poor strategy to reach Abuja targets and millennium development goals. Br Med J 2004;328:1129-32.

5 Eigege $A$, Kal E, Miri $E$ et al. Long lasting nets are synergistic with mass drug administration for interruption of lymphatic filariasis transmission in Nigeria. PLoS Negl Trop Dis 2013;7:e2508.

6 McGregor IA, Gilles HM. Diethylcarbamazine control of bancroftian filariasis; follow-up of a field trial in West Africa. Br Med J 1956;1 (4962):331-2.

7 Rebollo MP, Sambou SM, Brent T et al. Elimination of lymphatic filariasis in the Gambia. PLoS Negl Trop Dis 2015;9:e0003642.

8 Kelly-Hope LA, Molyneux DH, Bockarie MJ. Can malaria vector control accelerate the interruption of lymphatic filariasis transmission in Africa; capturing a window of opportunity? Parasit Vectors 2013;6: 39.

9 Nsakashalo-Senkwe M, Mwase E, Chizema-Kawesha E et al. Significant decline in lymphatic filariasis associated with nationwide scale-up of insecticide-treated nets in Zambia. Parasite Epidemiol Control 2017;2:7-14.

10 Boatin B, Richards FR. Control of onchocerciasis. Adv Parasitol 2006; 61:349-94.

11 Cochi S, Dowdle WR, eds. Disease Eradication in the $21^{\text {st }}$ Century; implications for Global Health. Strungman Forum Reports. Cambridge, MA: MIT Press, 2011; p. 319.

12 Dowdle WR, Hopkins DR, eds. The eradication of infectious diseases. Chichester: John Wiley \& Sons, 1998; p. 218.
13 Molyneux D. Eradication and elimination: facing the challenges, tempering expectations. Int Health 2015;7:299-301.

14 Molyneux D, Sankara DP. Guinea worm eradication: progress and challenges-should we beware of the dog? PLoS Negl Trop Dis 2017; 11:e0005495.

15 Gustavsen K, Sodahlon Y, Bush S. Cross-border collaboration for neglected tropical disease efforts-lessons learned from onchocerciasis control and elimination in the Mano River Union (West Africa). Global Health 2016;12(1):44.

16 Traoré S, Wilson MD, Sima A et al. The elimination of the onchocerciasis vector from the island of Bioko as a result of larviciding by the WHO African Programme for Onchocerciasis Control. Acta Trop 2009; 111:211-18.

17 Craig PS, Larrieu E. Control of cystic echinococcosis/hydatidosis: 1863-2002. Adv Parasitol 2006;61:443-508.

18 Katabarwa MN, Katamanywa J, Lakwo T et al. The Imaramagambo onchocerciasis focus in southwestern Uganda: interruption of transmission after disappearance of the vector Simulium neavei and its associated freshwater crabs. Am J Trop Med Hyg 2016;95:417-25.

19 Jacobson J, Bush S. Neglected tropical diseases, neglected communities, and conflict: how do we leave no one behind? Trends Parasitol 2018;34:175-7.

20 Molyneux DH, Hopkins DR, Zagaria N. Disease eradication, elimination and control: the need for accurate and consistent usage. Trends Parasitol 2004;20:347-51.

21 World Health Organization. Global programme to eliminate lymphatic filariasis: progress report 2016. Wkly Epidemiol Rec 2017;91:589-608.

22 World Health Organization. 2018. http://www.who.int/neglected diseases/news/Surveillance-presence-of-dracunculiasis-in-Angola/en/ (accessed 3 July 2018). 